result of chlorosis. One of these cases was seen in New York City. The woman had been sent to hospital with the diagnosis of an intracranial growth, and had suffered for a long time from constant headache and increasing loss of vision. She was unable when first seen to count fingers at a distance of a few inches. The possibility of an intracranial growth was negatived by the history and by absence of focal symptoms, and examination of the blood showed a moderate chlorosis, and this was thought to be a possible cause of the constant headache and the double-choked disc. She improved almost immediately on rest, baths and the administration of iron and bone marrow. Within six weeks vision was almost completely restored, and the headaches disappeared long before this. About one year ago I saw at my home a young girl with a severe choroiditis. Her hemoglobin was reduced to 32 per cent. For a year she had been anemic, and had been treated persistently by her father with many forms of iron and intestinal antiseptics, and by rest, but the anemia seemed to be increasing. I suggested the addition of bone marrow, and whether it was an accidental occurrence or not I can not say, but within a short time she had improved very markedly in every way, and the hemoglobin percentage had increased to 80 per cent.

Dr. W. E. Darnalr, Atlantic City, N. J.-So far as I have been able to observe these cases of chlorosis I have been impressed with the importance of the intestinal toxemia theory. Last summer an Irish girl of 22 entered the Atlantic City Hospital during my term of service. The hemoglobin was reduced to 20 per cent. Instead of the usual temperature of 100 or $101 \mathrm{~F}$. her temperature ran between 103 and $104.5 \mathrm{~F}$., which gave strong suspicions of the presence of some other disease. She was, of course, constipated. If she were allowed to go for one day without a movement of the bowel, her temperature, which might have been near normal, would suddenly rise to 104 or over. Repeated examinations of the blood were made to eliminate typhoid fever and malaria, and these were positively excluded. Strict attention was then given to the bowels, and an evacuation was secured regularly twice a day. As soon as this systematic treatment was established the temperature fell to normal, and in a comparatively short time she was completely restored to health, as shown by the blood examination.

Dr. WAHREk-I do not think it makes very much difference, therapeutically, what preparation of iron is selected, for no form of iron is absorbed into the system unless it has been converted into the chlorid. Then, why not give the chlorid? Well, it spoils the teeth, produces constipation and blackens the stools, and if long continued, interferes seriously with digestion. While we give peptonate of iron and many other forms, I wish to emphasize the importance of selecting one kind that seems best suited to the individual case. Our success with drugs depends very largely on our special experience with them. I made no special mention of eye symptoms, referring only to the pseudo-exophthalmic cases as an example. There are many cases described as chlorosis which probably should not be classified in this way; nevertheless this term presents a well-recognized symptom complex. I wish to say earnestly, in conclusion, that it is seldom that one finds two people who look precisely alike, and in the same way it is probable that there are no two people who possess exactly the same constitution. I believe every disease or disease complex is modified by this individuality. It is the business of the physician to study this point most carefully and treat the individual who is diseased, as well as the disease. Were this not true, we would need but very few drugs to treat such diseases as are amenable to drug therapeutics. Here again comes in that indefinable but sterling quality every good physician must have to insure success, namely, common sense, or good judgment, or whatever term is best to know it by.

Primary Tumors of the Heart.-Traina has collected in the literature thirty-six primary tumors of the heart. In order of frequency they were myxomata, fibrumata, and their combinations, myomata, pure sarcomata, lipomata, fibrosarcomata, angiomata and one chondroma. He describes in the Clinica Med. Italiana, xli, 2, a personal observation of a case of primary fibromyxoma.

\section{IS THE ADENOID OPERATION A JUSTIFIABLE SURGICAL PROCEDURE;}

AND, IF SO, SHALL IT BE DONE IN ACCORDANCE WITH THE RULES OF GENERAL SURGERY ?*

GEORGE L. RICHARDS, M.D.

FALL RIVER, MASS.

At first thought it would seem that to read a paper with this title before this Section is like raising up a man of straw and promptly proceeding to demolish it because it can make no resistance. I hope, however, to demonstrate that a paper with this title is neither untimely nor devoid of interest. The only logical answer to the query is an affirmative one; to which you will all mentally add, "We all operate that way"; and yet some of my observations have made me feel that in a good many instances this operation is not being done as though it were a justifiable surgical procedure, nor in accordance with the rules of general surgery.

Adenoid hypertrophy is a soft growth in the nasopharynx which interferes with nasal respiration and with the proper ventilation of the ear, and experience from the time of Meyer has taught it were better to remove it whenever symptoms of obstruction are present. Because they are easily reached for removal by operation-which operation must invariably be done in the dark-adenoids have frequently been removed in the most incomplete, and in many cases brutal, manner. And since they are done in the worst manner possible in many teaching clinies where Americans who are to be our future surgeons and specialists and members of our societies are taught, I am moved to relate to you some of my personal experiences in witnessing operations of this class done, not ten years ago, but so lately as a few months ago.

For many years German-speaking Europe was the great place for graduate study in the nose, throat and ear, on account of the wealth of material and the fame of the instructors; and of the audience before me probably the greater part have studied in German-speaking countries. As is well known to you all, the German is much more of an adept in the science of medicine and surgery, and especially in the pathology, than he is in the art; and at present most procedures are better done in America than abroad. It was, however, a surprise to me in revisiting a number of clinies to notice with what brutality, or at least so it struck me, the adenoid operation was done. While for obvious reasons I shall not give the names of the clinics or operators, the picture as presented is nevertheless an accurate one, and I have no doubt would be equally true of any number of teaching clinics.

Let me describe to you how I saw the adenoid operation done in five different foreign clinics, all of which have American students. In one the struggling child was taken in the arms of an assistant; the physician sat before the child, with an ordinary laryngeal mirror for a tongue depressor, without a mouth gag; the tongue was depressed, a Beckmann curette introduced behind the pharynx, one or two sweeps made, the child tipped forward and the operation was over. Plenty of blood came, but in the half dozen or more times that I saw the operation done in this way by this operator, and also by other operators in the same clinic, I saw no adenoids of any amount. The finger was not used to determine whether anything was left, nor was any at-

- Read at the Fifty-third Annual Meeting of the American Medical Association, in the Section on Laryngology and Otology, and approved for publication by the nxecutive Committee : Drs. Emil Mayer, C. R. Holmes and G. H. Makuen. 
tempt made to staunch the blood. The child shrieked loudly, was taken into an adjoining room, and the next child brought forward for operation.

In another clinic in the same city I saw the operation done in substantially the same way, only here the curette was used with such force that in one instance I saw a large portion of what looked like good healthy nasopharyngeal mucous membrane brought out. Here again no after-examination was made to determine whether or not the growth was removed.

In another clinic in another city I saw eighteen children operated on for enlarged tonsils and adenoids in one group. One after another they were seated around the room to shriek. and after they had all been operated on they were brought back for a second examination to see if any portions of adenoids were hanging in the pharynx or bits of tonsils remained. No cocain was used, but in this instance a tongue depressor and a mouth gag were employed, and the operations, while rapidly done, were more scientifically performed than in the two preceding instances.

In a fourth clinic the operation was done without anesthesia and with no attempt at mitigating the pain, yet from the surgical point of view aside from this I have no criticism to offer. Here I saw some twenty children operated on in one seance. While a nurse prepared a child for operation in one chair, the operator operated on another in another part of the room. There was good illumination with artificial light and a forehead mirror, and the instruments used were a most excellent mouth gag, tongue depressor and a Beckmann curette. The tongue was depressed, the curette skilfully used, and in three sweeps, one in the middle and one in each side, the growth was thoroughly removed, and an examination at once made with the finger to be sure that nothing was left. Here adenoids appeared in every instance. In the three preceding clinics I was always surprised at seeing how few adenoids were removed. As fast as the children were operated on they were all sent shrieking and spitting into an adjoining room; and you can imagine the pandemonium present when fifteen or twenty children, shrieking at the top of their lungs and spitting blood, were all assembled in one room. The skill of the operator, however, in this latter case admits of no question, and I am almost tempted to give his name.

In the fifth clinic, which was in England, adenoids were removed in the following manner: Nitrous oxid gas was administered by a skilled anesthetist, a nurse held the child in her lap, another nurse looked after the hands and feet of the patient, while the operator sat before the child, using a forehead mirror and artificial light. Anesthesia was usually complete in less than a minute, the tonsils were removed with the Mackenzie tonsillotome, and the adenoids with the Löwenberg curette followed by the Gottstein. The child's head was thrown forward, the bleeding was inconsiderable, the child was told to spit out the blood, and in about three minutes from the time of beginning the operation the child was able to walk into another room, where it was attended by a nurse until such time as it was able to go home, when it was given printed detailed directions for care for the next few days. For public clinic or hospital work this method seems ideal, since it is accompanied by an insignificant amount of blood as compared with an ether operation, is rapid and painless, and was used in this clinic for all but the very youngest children, whom it was deemed unsafe to anesthetize.
The methods in use in the first four clinics, which were large continental clinics, seemed to me absolutely brutal and incomplete; and I was told by a laryngologist of prominence that the operation itself had fallen somewhat into disrepute, as the growths frequently recurred. As you are all aware the German-speaking authors mostly advise the operation to be done without anesthesia, and now and then some of them claim that it is not painful. Even those who admit that it is painful seem to prefer to operate without anesthesia. Let me quote from several prominent authors: "The operation is painful without narcosis, and when very rapidly done can do harm to a very nervous child." But this author rather deprecates the necessity of employing narcosis, since, he states, the operation can be done in about three seconds. Another writer, an Italian, says: "I do not like narcosis, and only use it when I have children to operate on that can not be held rigidly enough, or when the parents object to its being done without. On the other hand, the pain is of so very short duration, and the operation lasts such a short time, that its risks ought not to be increased by the dangers of using narcosis." Another author states that the children do not realize much of anything until the operation is completed. and that good children have said after the operation that they have not suffered any pain. Furthermore, he states that the tissue contains few or no sensitive nerve fibers, and the child cries because he sees and feels the blood flowing out of the mouth and nose. $\mathrm{He}$ says that there are children so unruly, stubborn and obstinate that they can not be operated on except under narcosis. I noticed in my own observations that the children all cried much as they would have done in America; but I am glad to know that they cry, not from pain, but simply on account of the bleeding at the nose and mouth. There are signs of the dawning of a better day for these cases, as in a long monograph of recent date on adenoids, by a continental writer, anesthesia is spoken of as follows: "Local anesthesia is very incomplete and does not spare the child the psychic shock of the instruments and their introduction into the mouth. It is lymphatic, nervous, fearsome, delicate, sensitive children that have adenoids. The forcible holding, the light before the eyes, the large number of instruments, the forcing open of the mouth, the introduction of instruments into the pharynx, which is frequently too small for the instruments, the removal of portions of tissue-is all painful for the patient, as is also the scraping of the growth many times with the curette, which is per. haps not conformable to the pharynx wall, while the child is half suffocated with blood. This procedure frightens the child and causes a nervous shock. On the other hand, the operation is done more easily under anesthesia, and the danger of injury to neighboring structures is less." When I inquired personally among my continental friends as to why they did not use anesthesia for these operations they told me that the anesthesia was dangerous, the operation was short, the pain slight and it was not necessary. A friend of mine, of large private practice in one of the largest continental cities, told me. however, that he invariably used anesthesia, and frequently reoperated after the surgeons who did not regard anesthesia as necessary.

So much for operations across the sea. I am glad to state that, so far as I am aware, most American operators operate under anesthesia, and in a thorough manner. Yet no later than three years ago, in a paper read before this Association (I quote from the published proceedings, 1899), the finger-nail surgery was advocated 
as the proper thing for the removal of adenoids, and the writer stated: "I have never seen or rather felt a case of adenoma which was not entirely removable by the finger-nail, provided the latter was in its normal condition and had not been injured or broken. . . . If not all removed, either the same day or the next, if possible, I will again enter the cavity and, if necessary, with the steel finger-nail remove every bit of the diseased tissue discoverable. . . . Two days after, I examine the region, and if everything is well I re-examine after a further period of about three or four days, when, if the healing has been perfect, the upper pharynx will feel smooth and soft. and there will be no danger of recurrence." The steel finger-nail-an instrument much better suited to the dark ages than to twentieth-century surgery-was shown, which was to be used whenever the operator's finger-nail was out of condition. In justice to the members of the Section on Laryngology of 1899 , it must be stated that there was general dissent to the reader's proposition. I have also heard of other American surgeons who operate piecemeal for these growths.

The removal of the adenoid growth, if the growth is necessary to be removed at all, is an operation of moment to the person on whom it is done, and therefore should be done in as thorough a manner as possible, and with as little resulting shock. By the rules of general surgery as applied to this operation I mean: general anesthesia in children over two or three years of age, and in all adults in whom it is likely that cocain anesthesia will be insufficient, and proper preparation of operator and instruments, so as to minimize all the risks.

In older children and adults, where the nasopharynx is fully developed, the Schuitz adenotome or one of its modifications can be advantageously used under cocain or even without any anesthesia. It is especially adapted for use where the adenoid is mainly central. I have been pleased with it and I know that others also have. I do not think it is adapted for very young children, as in these the pharynx is hardly large enough or sufficiently developed for its successful use. In these, as above stated, I prefer general anesthesia and the ordinary instruments. This adenotome has not yet acquired very general usage. I have never seen it used in any clinic.

The question of the anesthetic to be employed, whether ether, chloroform, bromid of ethyl or chlorid of ethyl, is one that must be determined in accordance with the experience of the individual operator. In most cases I personally prefer ether. Rapid, but careful, deliberate operating should be the rule, the operator being neither in haste to finish in any given number of seconds nor unduly prolonging the operation; and sharp instruments should be used, so that as much as possible the growth may be cut away rather than torn away, so as to leave a smooth cavity. The removal of the adenoids, not of all the structures to the spinal column, is what we desire. The pharynx ought to be covered with mucous membrane and not with fibrous connective tissue which will be a source of dry catarrh during the remainder of the life of its owner.

During the operation I believe in staunching the blood with tampons, since I see no reason for allowing the patient to lose any more blood than is necessary, although $I$ read in all the books that staunching the blood is unnecessary.

Before regarding the operation as finished, determine by examination that the growth has been entirely removed. Keep the child in bed for a day or two following the operation, and do not feel in his nasopharynx at frequent intervals thereafter. If the operation was satisfactorily done no after-examination is necessary.

For the preliminary finger examination previous to operation and for post-operative examinations protect the finger with the rubber finger cot. The details of the operation, preparation, position, asepsis, instruments, etc., do not come within the scope of this paper.

Fortunately, unpleasant sequelæ in the way of sepsis or hemorrhage are almost unknown, considering the number of adenoid operations which are performed. I must, however, considering how difficult it is to properly disinfect or sterilize one's finger nail, protest against its use as a surgical instrument in these cases.

\section{DISCUSSION.}

DR. L. C. ClINE, Indianapolis-Two or three years ago I was severely criticised by some of the members of this society because I advocated doing this operation without an anesthetic. I believe the use of anesthetics is overdone in this operation, and only in the exceptional case is an anesthetic necessary. In the majority of cases as good results may be obtained without the use of an anesthetic, and we must admit that there is a danger in the use of anestheties. Only a short time ago there were 18 or 19 deaths reported from anesthesia, and I am sure anesthesia is not without danger in this operation. We have all learned certain ways of doing operations, and when we become skilled in doing an operation in a certain way, that is the right way for us to do it. Other men may have as good success by some other procedure. My custom is, in the majority of cases, to have the child firmly held by an assistant. I do not have a great array of instruments to show the patient. I do not allow the parents to speak to the child about what I am going to do. I pick up my Gottstein instrument or a moditication of it, and before the child realizes what has happened 1 have done my work. They cry a little afterward, but I would rather they would cry than to have to perform artificial respiration to bring them to after the use of an anesthetic. I have had that experience. We have to adapt our methods to the individual case, I think, and we are not justified in subjecting every case to an anesthetic. I would not use it in my own child, but I would treat it without an anesthetic unless an anesthetic seemed necessary. I believe a good, reliable, efficient operation can be done without even local anesthesia. Such a procedure may be described as brutal and all that, but if the adenoids are soft you can operate without much pain.

Dr. J. H. MacdonalI, New York City-This operation is now a great deal in disuse and disrepute by the operators that have done it. The same preparations should be taken for it as for any other operation in surgery. The urine should be examined, the general conditions of the patient should be looked into, and the nervous condition of the patient and the age should be taken into consideration. Many of our clinics do not examine the urine, and do not even know whether the bowels are loaded, as happened in a case related by Dr. Cline. All these things are overlooked in what is considered the little operation for the removal of adenoids. This operation should be done with the proper technic, with the aid of nurses and intelligent assistants. There should be no struggling. Then the use of anesthesia would not be in disrepute. In regard to the choice of an anesthetic, I have, with Dr. Gleitzmann of New York, used bromid of ethyl in over 500 cases without a death. I have used the A. C. E. mixture and also chloroform. We are careful in the selection and the use of an anesthetic. We take the condition of the blood into consideration. We see that the child is thoroughly relaxed, that is, of course, in children over two years of age and who are not of a nervous temperament. That has been the teaching of Dr. Gleitzmann while I have been his assistant for over five years. In regard to ether, there is an unknown quantity. But if we go into the subject with the same care as if we were to do a laparotomy, there would not be half the deaths.

Dr. Sargent F. Snow, Syracuse, N. Y.-I agree with Dr. Richards that this operation should be done as a surgical pro- 
cedure. For about three years I have been trying the use of bromid of ethyl. I have also used other anesthetics, such as ether and chloroform and laughing gas. I must say that if I can have an assistant to hold the patient, who is famillar with the work and an anesthetist who is familiar with giving bromid of etriyl, I prefer it to all other anestheties. With bromid of ethyl we get anesthesia in from 90 to 120 seconds, and sometimes in 60 seconds sufficient to amputate both tonsils and remove the adenoids. Almost immediately afterward our patient is conscious, with no sickness and with very little hemorrhage. This anesthetic is most excellent if the drug be fresh and our assistants acquainted with their work. The laughing gas in my hands so far has not proved all I wanted. The anesthesia I get from it is not sufficient. Perhaps my anesthetist has not pushed it far enough, but we do not get sufficient relaxation. I do like the patient in an upright position, which seems safe when we use bromid of ethyl.

Dr. G. McAuliffe, New York City-I did not understand from Dr. Richards' paper whether or not he staunched the bleeding. As soon as the adenoid tissue is all out, I have not witnessed any bleeding.

Dr. Richard-I did not give the details of the operation, but my plea was for good surgery. The current number of the International Journal of Surgery contains an article in which my own views are stated.

\section{THE DIAGNOSIS OF CARCINOMA OF THE LARYNX.*}

OTTO T. FREER, M.D. CHICAgo.

It is unfortunately true that ordinarily carcinoma of the larynx is not discovered until nothing but total laryngectomy offers the patient a chance for existence. One of the reasons for this is the fact that the diagnosis of the affection is often difficult and may be impossible for even an expert. Another cause for the lateness of recognition of the disease is the slight regard usually paid by the patients to the persistent hoarseness that may precede the graver manifestations for years as the only symptom. A third and avoidable reason for the unrecognized advance of malignant laryngeal tumors is the widespread indifference to the acquirement of even moderate skill in laryngoscopy shown by a great part of the profession. Many physicians treat patients with chronic hoarseness by general means and do not even inspect the larynx. I have known a patient to be sprayed for six months for supposed laryngeal catarrh by means of an atomizer and tongue depressor while his unseen carcinoma rapidly advanced to a hopeless stage. In another instance the laryngoscope was not used until the carcinoma had penetrated the laryngeal cartilages and appeared as a tumor in the neck. A glance at the literature of laryngectomy will show how late carcinoma of the larynx usually comes to the operator. Chronic hoarseness, especially in men past forty should make a laryngoscopic examination an imperative duty to all practitioners.

There are no general conditions with the exception of age and sex that are of use in determining the existence of carcinoma. Until the disease has advanced to the stage of dysphagia, extensive ulceration, sloughing and perichrondritis, it does not interfere materially with health.

Though most of the patients affected with the disease have passed their fortieth year, a large proportion of cases is seen before this period and even childhood is not exempt. This is shown by the statistics of Send-

* Read at the Fifty-third Annual Meeting of the American Medical Association, in the Section on Laryngology and Otology, and approved for publication by the Executive Committee: Drs. ziak containing 479 cases of carcinoma of the larynx and that of E. Kraus with 187 cases, a total of 666 . Five of these were found in the two first decades of life, 26 between the 20 th and 30th year, 59 between the 30 th and 40th, 170 between the 40 th and 50th, 264 between the ages of 50 and 60,110 between 60 and 70,15 between 70 and 80.

Sex is of importance in determining the existence of laryngeal carcinoma, as the statistics of numerous observers show that men are from six to seven times more liable to the disease than women.

None of the symptoms of carcinoma are of much value in proving its presence, but they are of great use in arousing suspicion of its existence. In the majority of cases hoarseness is the sole evidence of commencing malignant disease for a long period, generally from one to three years. Chiari mentions a case in which hoarseness lasted nine years before other symptoms made their appearance and voice disturbance in his other cases extended over an average of two years before there was a change in the manifestations. This long period of comparative latency of the disease gives abundant opportunity for conservative operative interference and serves to differentiate carcinoma from the rapidly destructive laryngeal lesions of syphilis and tuberculosis.

The symptom of pain appears in the later stages of carcinoma, characterized by sloughing and ulceration. It is common to all the ulcerative affections of the upper larynx and therefore not peculiar to malignant disease though in some cases a prominent feature. It serves to exclude the benignant growths from consideration as these are never associated with pain. In carcinoma this generally takes the form of otalgia, occurs during swallowing or sometimes spontaneously and darts from the larynx to the ear, angle of the jaw or back of the head. Fraenkel states that it always appears in the ear corresponding to the side of the larynx affected, and that he has never found pain as a symptom of carcinoma where the epiglottis or aryteno-epiglottic folds were not ulcerated. Ulceration of the cords did not, in his experience, give rise to otalgia. Delavan regards lancinating pain as an important symptom, but his views are not shared by the majority of authors, who agree that it is usually a late and inconstant manifestation. Scheier found in 102 cases of carcinoma only 9 who complained of otalgia or pain radiating to the back of the head. In my experience pain is not a regular or prominent feature of laryngeal carcinoma.

The dyspnea of carcinoma ordinarily lasts many weeks or months before it becomes dangerous, but if perichondritis or other acute inflammatory complication occur it may come on suddenly, and whether its onset be fast or slow it almost invariably demands tracheotomy. A history of slowly increasing laryngeal stenosis in an old man is very suggestive of carcinoma. The stench that carcinoma gives to the breath is a characteristic but very late symptom, which may also occur in syphilitic necrosis of the cartilages.

It is thus evident that the patient's general state and symptoms offer but slight aid in making a diagnosis and that dependence must rather be placed on laryngoscopic and microscopic examination.

It is customary to divide carcinomata of the larynx into intrinsic ones; those originating on the vocal cords, ventricular bands, in the ventricle or inter-arytenoid or subglottic regions and extrinsic carcinomata; those seated on the epiglottis, aryteno-epiglottic folds, in the fossa pyriformis and on the posterior surface of the cricoid ring. Observers agree that carcinoma has its origin 